\title{
LEVERAGING CLOUD-BASED SERVICES AND TOOLS FOR CURRICULUM AND ENGINEERING ATTRIBUTES MANAGEMENT
}

\author{
Guy Bégin \\ Director of microelectronics engineering, Département d'informatique/UQAM \\ P.O. Box 888, Station Centre-Ville, Montréal H3C 3P8 \\ begin.guy@uqam.ca
}

\begin{abstract}
Canadian engineering schools must make the transition to outcome-based programming, assessment, and accreditation. The task can be daunting, especially for small schools or programs that cannot rely on extensive information technology support.

Cloud-based services are a quick, low-cost option for facilitating many data-management and collaborative tasks required by the process. Cloud services have evolved from mere on-line storage to the "software as a service" paradigm.

We report our experience with two services that facilitate collaborative work. Using on-line, specially crafted questionnaires, information may be automatically collected and formatted into spreadsheets, providing a powerful, general purpose data collection engine. This approach was used at various stages in the transition to graduate attributes processing: curriculum mapping, assessment, etc. On-line services have also been used to create a distributed repository of relevant literature for supporting the work of the "attributes" team.
\end{abstract}

\section{Keywords: Cloud, On-line, Collaborative, Workflow,} Engineering, Education, Graduate, Attributes

\section{INTRODUCTION}

Canadian faculties and schools of engineering are required to make the transition to outcome-based programming, assessment, and accreditation to meet the new Canadian Engineering Accreditation Board (CEAB) Graduate Attributes requirements [1]. The task can be daunting, especially for smaller schools and/or programs that cannot rely on extensive administrative and information technology support. Even though UQAM is, by Canadian standards, a large university, it offers a single accredited engineering program, a bachelors of microelectronics engineering [2], and the resources allocated to the transition process are limited.

The re-engineering of programs entails a number of tasks, to be performed by a variety of actors: professors, instructors, administrative staff, etc., who will undoubtedly argue they have more important things to do. While a committed "attributes" team is a prerequisite, coordinating and rendering the work as effortless as possible for everyone involved is key to a successful experience.

Early on, cloud-based services [3] were identified as a quick, low-cost option for facilitating many of the datamanagement and collaborative tasks required in this process. Previous experiments in a different context had already convinced us of the value of sharing workloads efficiently using collaborative tools. This paper presents our experience with using cloud-based services to facilitate the data management activities associated with the evolution of our engineering program towards the new CEAB objectives.

After a general presentation of collaborative environments in section 2 and cloud services in section 3 , we recount our experience with two cloud providers in section 4, discuss the issues in section 5 and offer concluding remarks in section 6 .

\section{COLLABORATIVE ENVIRONMENTS}

Web technology and electronic networks have created a novel work environment where the constraints of location and time can be escaped. With the support of network-based collaborative tools that provide communication, coordination and cooperation facilities, a virtual team may be assembled and put to work across time and space [4].

Collaborative computing is defined as the use of computers to support coordination and cooperation of two or more people who attempt to perform a task or solve a problem together [5], [6].

Although collaborative systems are often viewed as a way to bring together widely dispersed teams, local teams can also benefit from improved communication and organization.

Based on when and where the interaction takes place, different kinds of collaborative systems may be distinguished. Our needs focused on workflow facilitating and collaborative writing systems, which belong to the different-time/different-place category. 
Setting up a shared, web-based workspace specifically for a collaborating team might seem like unnecessary additional effort, but this is where cloud technologies provide an interesting option.

\section{CLOUD SERVICES}

According to NIST, cloud computing is a model for enabling ubiquitous, convenient, on-demand network access to a shared pool of configurable computing resources that can be rapidly provisioned and released with minimal management effort or service provider interaction [3].

Various service models are possible, depending on how much of the processing and infrastructure is delocalized in the cloud. In cloud Software as a Service (SaaS), applications running on a cloud infrastructure provide services to the consumer. In cloud Platform as a Service (PaaS), consumer-created or acquired applications are deployed onto the cloud infrastructure. Finally, with cloud Infrastructure as a Service (IaaS), the consumer is able to deploy and run arbitrary software, including operating systems and applications, in the cloud.

\subsection{Selecting Cloud Service Providers}

Cloud services are basically available through a Web interface. Many providers also provide a dedicated client software for interfacing with popular platforms. Typically, data storage and synchronization are part of the basic service. The number of users, amount of storage and/or processing power and degree of customizability is the basis for services grades and subscription levels. Free, advertising-supported and paid accounts are offered.

Not all cloud providers offer services facilitating collaboration. The basic requirements for our usage were easy access using a variety of client platforms, flexibility, the ability to support ISO standard document formats for office applications (OpenDocument) [7] (for both opening and exporting), and inter-operation with other de facto documents standards (e.g., Microsoft formats) for integration with traditional technologies. Finally, data lock-out, where all of the information (and the effort put into creating it) is tied to one provider or a proprietary format and cannot be easily extracted or converted should be avoided as much as possible.

\subsection{Google Cloud Services}

Google offers a constantly evolving range of service packages, including Gmail, Calendar, Talk, Sites, Drive and Groups. The last two were found to be especially well suited to our purposes. The free 5GB of storage provided ample capacity for the needs of this project.
Formerly known as Google Docs, Google Drive [7] offers a number of free, customizable SaaS tools, providing notably an on-line office suite. Using a simple internet browser as a client platform, text documents, spreadsheets and presentations can be created, shared and edited by multiple users simultaneously, with updates in real time. Files are automatically saved. The office suite supports a large variety of formats (ODF, HTML, PDF, RTF, Text, Microsoft Office), and a revision history is provided. Users may add and reply to comments on anything (PDF, image, video file, etc.), with notifications when other people comment.

Access control is customizable to a certain extent, with the ability to make a file public on the web, allow anyone with the link to access it, or keep it private. Files and folders belong to owners, who may grant permission to edit, view or comment them to others. In order to facilitate access management, one can create a Google Group for the sole purpose of regrouping users. Access to a resource may then be granted to a whole group as if it were a single user. Single sign on authentication is convenient: once a user is authenticated to one Google service, all the others are automatically available.

\subsection{Mendeley}

Mendeley [8] is an on-line service for sharing and managing research papers, available as a basic free version with $2 \mathrm{~GB}$ of free web storage space, and also in premium payable versions. It interfaces via a Web interface or using a dedicated client software. Mendeley manages a library of papers in PDF format. Basic citation data is automatically extracted from the metadata of the PDF and has to be stored on the user's computer; locally storing copies of the documents themselves is at the user's discretion, on a file by file basis. Full-text search is possible across the whole document library.

For collaborating, private and public groups with shared libraries may be created. A PDF viewer supporting sticky notes and text highlighting is integrated with the desktop client. When a group member adds a note, highlight or summary to a group document, the edit is visible to all the members of the group, in real time.

\section{OUR CLOUD EXPERIENCE}

\subsection{Google Forms}

One tool that has proved useful at many stages of the evolution towards outcome-based programming is Google Forms. It allows one to create customized surveys or questionnaires that are accessible on line. The information entered is collected and may be automatically connected to a spreadsheet populated with time-stamped questionnaire responses. It is possible to 
control the form's page-to-page navigation based on responses and form answers may be pre-populated.

Forms has been used as a general purpose data collecting engine for several tasks. For assisting in mapping the graduate attributes within the curriculum, a special purpose form has been created. The resulting questionnaire, shown in Fig. 1, is used to collect information on where graduate attributes are developed and/or evaluated within the different activities of the curriculum. By simply answering the on-line questionnaire once for each course or activity considered, each instructor was able to document the attribute-related information for the courses under his responsibility. Data collected was automatically aggregated and centralized into a spreadsheet, as shown in Fig. 2. The spreadsheet was shared with the "attributes" team for further manipulation and processing.

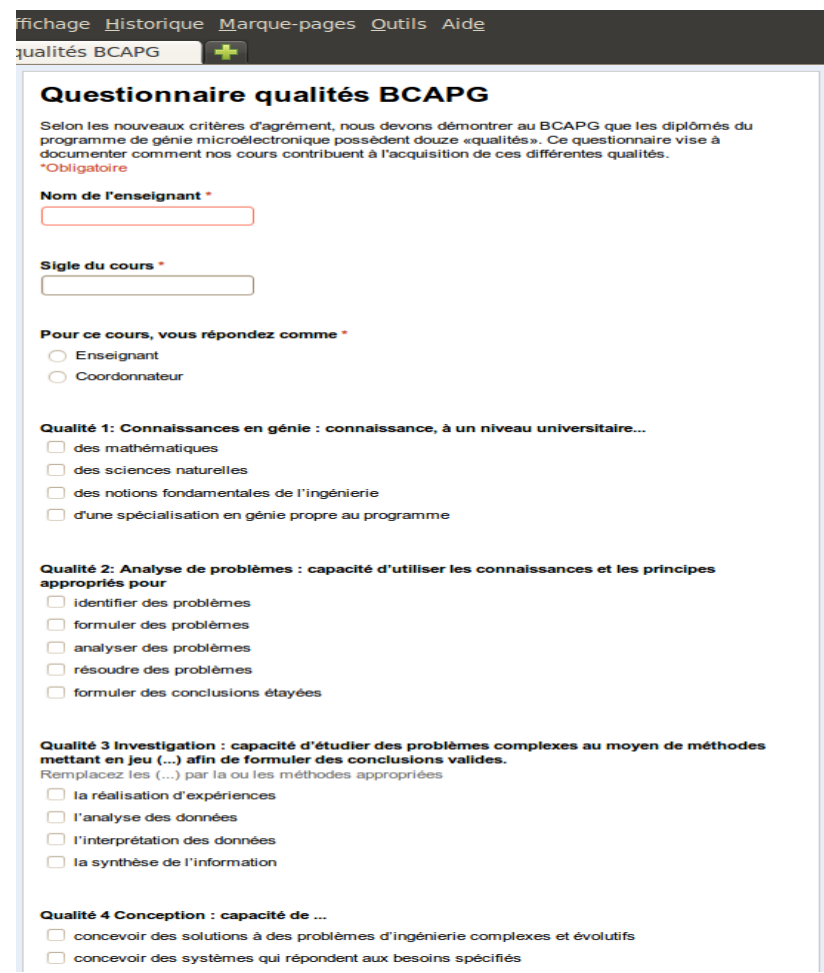

Fig. 1. Curriculum survey form.

In order to keep track of the progress of the curriculum mapping data gathering, a spreadsheet listing all the activities of the program was created and shared with all the participating respondents. After having completed the curriculum survey form for one course, a respondent would update the status for this course in the spreadsheet. Figure 3 shows a snapshot of the list at a certain point in the process, with activities on the left, and respondent names on the right. Each participant, as well as the "attributes" team, could therefore monitor progress in real time. Percentages of completion could also easily be obtained.

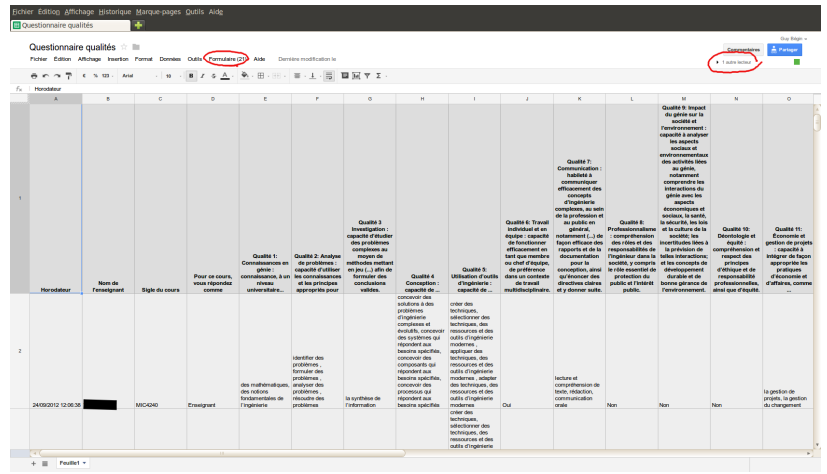

Fig. 3. Survey responses aggregated in a spreadsheet.

Once the questions and basic organization of the questionnaire are established, creating the form itself is a straightforward process. For example, the form of Fig. 1 was put together in less than an afternoon. More complicated forms with flow control based on responses require only a bit more planning.

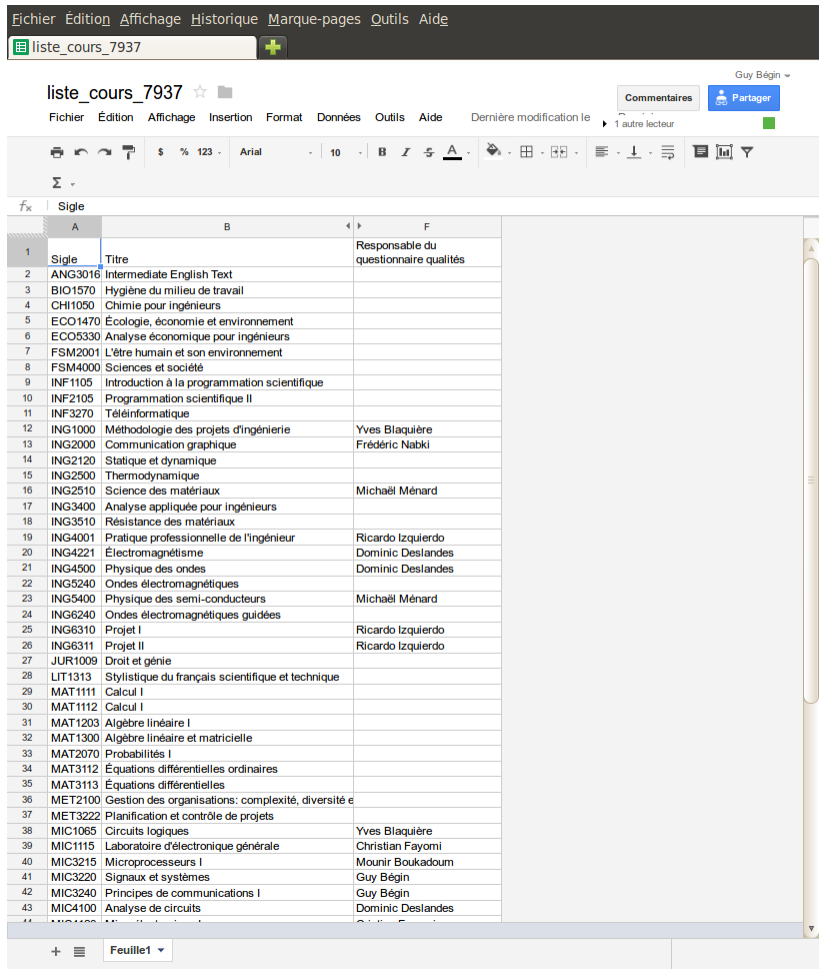

Fig. 3. Curriculum mapping progress.

Google Forms can also be used to support the assessment process, a key element in an outcome-based program. Forms have been created to facilitate the use of evaluation rubrics, such as the simple one shown in fig 4. The on-line forms can be used by evaluators (instructors, internship supervisors, etc.) to directly enter their assessment of students or interns performance, from anywhere. As seen on Fig. 4, the descriptive levels are 
prepended with numeric scores, which may be readily extracted by a simple operation in the corresponding spreadsheet, as shown in the rightmost column on Fig. 5. Further processing (computing totals, averages, etc.) can therefore be automated very easily. Once again, the data collected may be centralized, which facilitates programwide consolidation of information.

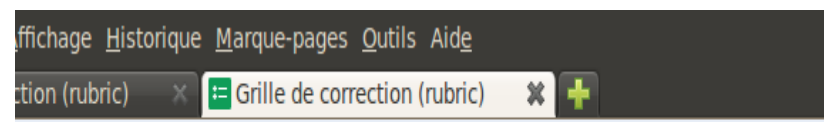

\section{Grille de correction (rubric) \\ Utilisation de l'équipement de manière à ne pas mettre en danger l'équipement ou les circuits quI lui sont connectés \\ 1 Dommages aux circuits ou aux équipements en raison d"un usage incorrect \\ 3 Certains des risques pour les circuits ou les équipements en raison d'un usage incorrect \\ 5 Utilisation sécuritaire des circuits et des équipements \\ 7 Précautions de sécurité allant au-delà des exigences

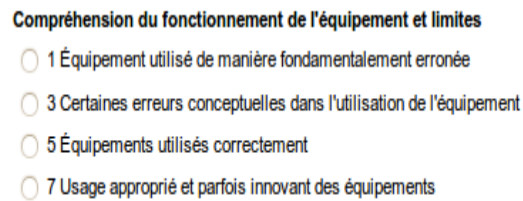 \\ Envoyer \\ Nenvoyez jamais de mots de passe via l'outil Formulaires Google. \\ Fourni par Google Documents}

Fig. 4. Rubric form.

\subsection{A Mendeley Repository}

Mendeley has been used to create a repository of relevant literature for supporting the work of the "attributes" team. A dedicated group was setup, where research papers relevant to the transition process are collected and commented as the work progresses. This way, all members of the team can stay in sync with regards to documentation.

The library may be organized using folders. However, since the access control mechanisms of Mendeley apply at the level of groups, and not at folders, it was not directly possible to create a subset of selected papers to be be made available for consultation only to every participant involved in the transition process. This limitation may be circumvented by creating a separate public group and duplicating content into it from the original group.

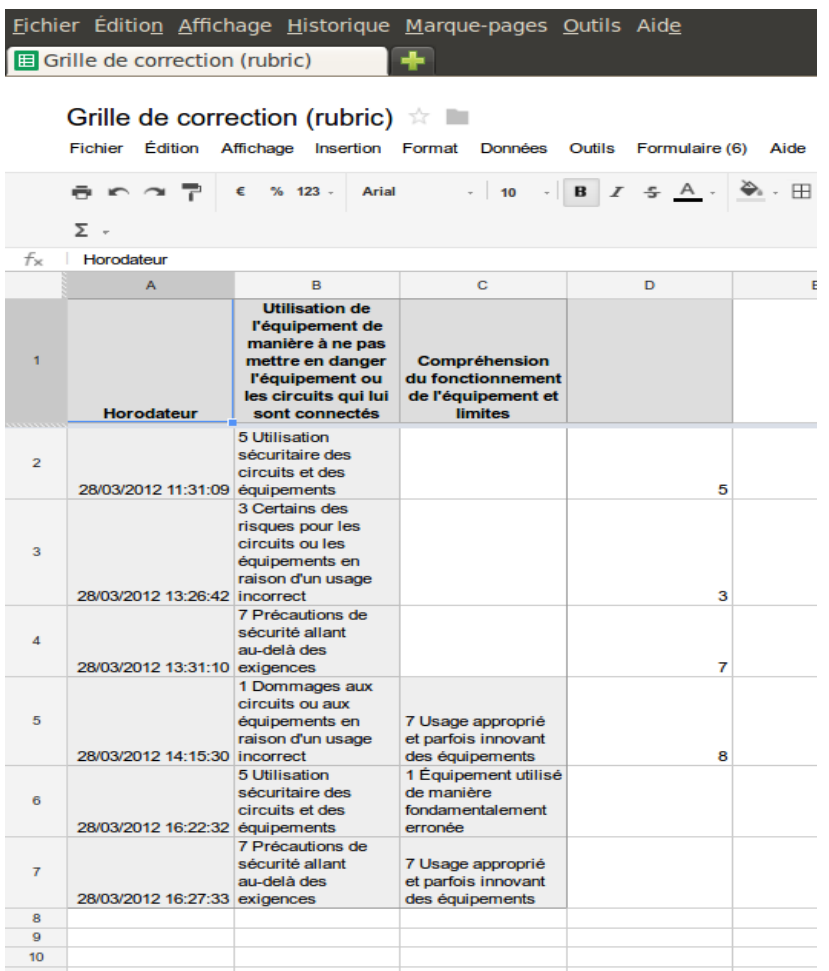

Fig. 5. Evaluation spreadsheet.

\section{ISSUES}

Our experience with free cloud SaaS has generally been positive. There are nonetheless certain limitations and challenges that have to be considered. The amount of storage and file size limitations could become an issue with time and usage. Upgrading to a paid account is the natural solution.

Security is another area where open questions remain. The confidentiality, integrity and availability of information hosted in the cloud should not be assumed. Google maintains that all data hosted remains secure, and that availability is close to $99,9 \%$, but such claims are all but impossible to verify.

On the other hand, by accepting Google Drive's terms of service [9], we grant Google with

"...a worldwide license to use, host, store, reproduce, modify, create derivative works (such as those resulting from translations, adaptations or other changes we make so that your content works better with our Services), communicate, publish, publicly perform, publicly display and distribute such content."

However, the users keep "...ownership of intellectual property rights..." In short, Google can do pretty much anything with the content except pass it off as its own.

The decision to leave important amounts of data solely in the hands of a third party, however reputable, should not be taken lightly. High-tech companies come and go, and even acquisitions turn out to have an impact on the attitude towards openness. The recent acquisition 
of Mendeley by giant journal publisher Elsevier [10], decried by many, is a reminder that things can evolve in unpredictable ways in the future. One way to play safe in the long term is to look to third party providers that offer back-up and account recovery services [11], [12].

Data lock-out is an issue that tends to go unnoticed until one wants to move away from a service. With Google Drive, documents, spreadsheets, etc. may all be downloaded and saved locally with a choice of formats. However surveys created with forms, being a special functionality of the spreadsheet application, cannot be recovered or saved in any way.

The granularity of access control of the cloud services we tested is somewhat coarse. Even with our relatively simple usage scenarios, we have had to resort to a few tricks to be able to do what was needed.

Finally, at the risk of stating the obvious, be reminded that network access a prerequisite for all theses services.

\section{CONCLUDING REMARKS}

Our experience with the free version of cloud services has convinced us of the value of collaborative interaction supported by cloud services. It is significantly easier to stimulate the participation of team members when a good portion of interactions can be liberated from constraints of time and place.

Although we have not yet reached the limit of the free offerings, it is worthwhile to envision what the next stage would be. The obvious option would be to go with paid version of the services we have used. The next level with Google is Google Apps, a paid account service providing independently customizable versions of several Google products under a custom domain name. A special version, Google Apps for Education [13], is available for schools, colleges, and universities. Of course, the security issues mentioned in section 5 remain.

Other options are more involved but ensure a better control over data and security in general. Alfresco [15] is a free/libre enterprise content management system for documents, web, records, images, and collaborative content development. Although not a cloud service per se, installing and using Alfresco is akin to becoming one's own cloud provider.

If the idea of running your own cloud is appealing, OwnCloud [16] is a free/libre software alternative to commercial cloud providers. Compared to commercial offerings, services are somewhat limited: file storage, synchronization, sharing of content across groups, document viewing for OpenDocument documents (within the web browser), bookmarking, as well as shared calendar, task scheduler and address book. The number and variety of applications supported is likely to improve in the future.

Whatever the approach, web based collaborative tools will certainly be part of the solutions that will lead us to an improved engineering program.

\section{References}

[1] CEAB, Accreditation Criteria and Procedures Report 2012, Available as of April 24, 2013 from http://www.engineerscanada.ca/files/w_Accreditation_Crit eria_Procedures_2012.pdf

[2] http://www.micro.uqam.ca

[3] Peter Mell, Timothy Grance, "The NIST Definition of Cloud Computing Recommendations of the National Institute of Standards and Technology," Nist Special Publication, vol. 145, no. 6, p. 7, 2011.

[4] Georgia Bafoutsou, Gregoris Mentzas, "Review and functional classification of collaborative systems," International Journal of Information Management, vol. 22, no. 4, pp. 281-305, Aug. 2002.

[5] Nathanial S. Borenstein, "Computational mail as network infrastructure for computer-supported cooperative work," in Proceedings of the 1992 ACM conference on Computer supported cooperative work CSCW 92, 1992, no. November, pp. 67-74.

[6] Eve. M. Schooler, "Conferencing and collaborative computing," Multimedia Systems, vol. 4, no. 5, pp. 210 225 , Oct. 1996.

[7] ISO/IEC 26300:2006/Amd 1:2012 - Open Document Format for Office Applications (OpenDocument) v1.1, Available as of April 24, 2013 from http://www.iso.org/iso/home/store/catalogue_tc/catalogue detail.htm?csnumber $=59302$

[7] http://googleblog.blogspot.ca/2012/04/introducing-googledrive-yes-really.html

[8] Mendeley, http://www.mendeley.com

[9] Google Drive Terms of Service, Available as of April 24, 2013 from http://support.google.com/drive/bin/answer.py? $\mathrm{hl}=$ en\&answer $=2450387$

[10] http://www.elsevier.com/about/pressreleases/corporate/elsevier-acquires-mendeley,-aninnovative,-cloud-based-research-management-and-socialcollaboration-platform

[11] Cloudlock.com, http://www.cloudlock.com

[12] Backupify, https://www.backupify.com/products/googleapps-backup

[13] Google Apps for Education, http://www.google.com/enterprise/apps/education

[14] OwnCloud, http://owncloud.org

[15] Alfresco, http://www.alfresco.com 